\title{
MODELING OF ACOUSTIC FIELD FOR A PARAMETRIC FOCUSING SOURCE USING THE SPHEROIDAL BEAM EQUATION
}

\author{
Lili Yu ${ }^{\mathrm{a}}$ \\ Junhua $\mathrm{Li}^{\mathrm{b}}$

\section{Chaojian Shi ${ }^{\mathrm{a}}$} \\ a) Department of Navigation Technology, Shanghai Maritime University, \\ Shanghai, China \\ b) College of Ocean Science and Engineering, Shanghai Maritime University, \\ Shanghai, China
}

\begin{abstract}
A theoretical model of acoustic field for a parametric focusing source on concave spherical surface is proposed. In this model, the source boundary conditions of the Spheroidal Beam Equation (SBE) for difference frequency wave excitation were studied. Propagation curves and beam patterns for difference frequency component of the acoustic field are compared with those obtained for Khokhlov-Zabolotskaya-Kuznetsov (KZK) model. The results demonstrate that the focused parametric model of SBE is good valid for a large aperture angle in the strongly focused acoustic field. It is also investigated that high directivity and good focal ability with the decreasing of downshift ratio and the increasing of half-aperture angle for the focused parametric model of SBE.
\end{abstract}

Keywords: parametric focusing source, difference frequency wave, SBE, acoustic field

\section{INTRODUCTION}

Since the difference frequency waves generated by parametric array have low frequency, high directivity and resolution, and good penetrative performance, parametric array is applied for underwater detection $[2,6]$. There is a great significance for distinguishing seabed properties and detecting buried object $[1,5]$. The experimental results for the near field of a parametric planar source have been published in Ref.5, which have carried out computations of the near field on the basis of the Khokhlov-ZabolotskayaKuznetsov (KZK) equation, and favorable agreement between the theoretical and experimental results was obtained [9]. The nonlinear behavior of a bounded sound beam generated by a parametric planar source in the far-field region of the source was also studied in Ref.6, which have introduced a coordinate transform in order to accommodate the spherical spreading of the acoustic field [4]. However, these works were generally used to describe nonlinear interactions of a parametric planar sound source. Although effects of focusing on the parametric interaction between two focused beams generated by monochromatic sources was investigated with KZK equation, this model is more suitable for a weak focused ultrasound beam because that it derived under the paraxial approximation and the upper limit of the applicability is about for the half aperture angle [3].
Later, the spheroidal beam equation (SBE) proposed by Kamakura et al. [10] used the oblate spheroidal coordinate system for theoretical prediction on strongly focused ultrasound beams with a circular aperture; the upper limit of the half aperture angle is up to $40^{\circ}$. Moreover, SBE can be easily extended to the analysis of nonlinear harmonic generation with high frequencies in finite amplitude sound beams [8]. However, SBE is generally used to describe sound propagation for geometrical self-focusing source with a single-frequency wave excitation. Currently, there are few reported results referring to SBE application in nonlinear interactions for difference frequency waves generated by parametric focusing source.

In this paper, we study some boundary conditions for difference frequency waves on a strongly focusing source using SBE. A strongly focusing source radiates a difference frequency sound wave, and some modifications are made to allow a boundary condition with two prescribed frequencies. In this case, two sinusoidal waves at the source are assumed to be just adjacent harmonics. The fundamental component corresponds to difference frequency sound. The numerical results are compared with results obtained in weakly focused amplitude sound beams, where KZK model have been used. Then, the propagation curve and beam patterns of difference frequency wave for SBE are investigated by 
varying parameters such as downshift ratio and halfaperture angle.

\section{THEORY AND METHODS}

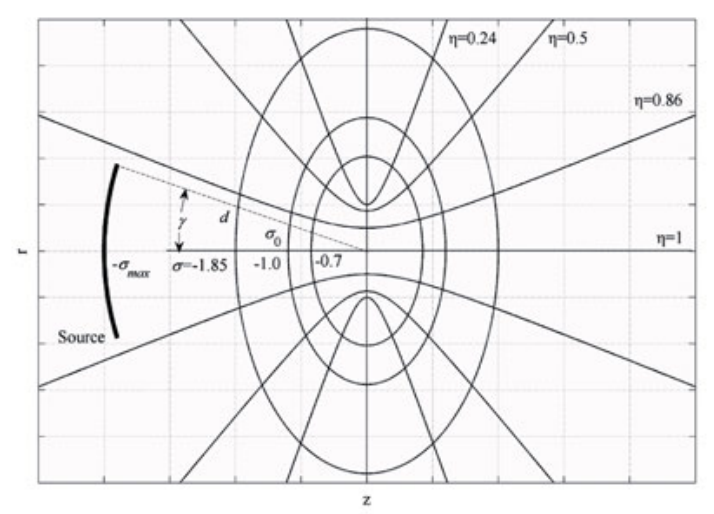

Fig.1. Oblate spheroidal coordinate system

To explain sound propagation with a large aperture angle, we apply SBE in an oblate spheroidal coordinate system [10] (Fig. 1). The focused ultrasound field is divided into two regions, i.e., $\boldsymbol{\sigma}<\boldsymbol{\sigma}_{0}$ and $\boldsymbol{\sigma} \geq \boldsymbol{\sigma}_{0}$, where $\boldsymbol{\sigma}_{0}$ denotes a specific transition location. The former division near the source is a spherical wave region, and the latter division near the focus is a plane wave region. The SBE model to describe the spheroidal and planar waves in two regions are expressed as follows respectively [10]:

$$
\begin{aligned}
& \frac{\partial^{2} \bar{p}}{\partial \tau_{s} \partial \sigma}+\frac{1}{2} \frac{\sin 2 \theta}{\sigma\left(1+\sigma^{2}\right)} \frac{\partial^{2} \bar{p}}{\partial \tau_{s} \partial \theta}+\frac{\varepsilon \sqrt{\sigma^{2}+\sin ^{2} \theta}}{\sigma\left(1+\sigma^{2}\right)} \times\left(\frac{\partial^{2} \bar{p}}{\partial \theta^{2}}+\cot \theta \frac{\partial \bar{p}}{\partial \theta}\right) \\
& +\frac{E}{\sigma} \frac{\partial \bar{p}}{\partial \tau_{s}}=-\frac{\sqrt{\sigma^{2}+\sin ^{2} \theta}}{\sigma}\left(\alpha b \frac{\partial^{3} \bar{p}}{\partial \tau_{s}^{3}}+\frac{b}{2 l_{D}} \frac{\partial^{2} \bar{p}^{2}}{\partial \tau_{s}^{2}}\right) E \quad\left(\sigma<\sigma_{0}<0\right) \\
& \frac{\partial^{2} \bar{p}}{\partial \tau_{p} \partial \sigma}-\frac{\sigma}{1+\sigma^{2}} \sin \theta \frac{\partial^{2} \bar{p}}{\partial \tau_{p} \partial \theta}-\frac{\varepsilon(2-\cos \theta)}{1+\sigma^{2}} \times\left(\frac{\partial^{2} \bar{p}}{\partial \theta^{2}}+\cot \theta \frac{\partial \bar{p}}{\partial \theta}\right) \\
& =\left(\alpha b \frac{\partial^{3} \bar{p}}{\partial \tau_{p}^{3}}+\frac{b}{2 l_{D}} \frac{\partial^{2} \bar{p}^{2}}{\partial \tau_{p}^{2}}\right) E \\
& \left(\sigma \geq \sigma_{0}, \sigma_{0}<0\right)
\end{aligned}
$$

where the variables $(\boldsymbol{\sigma}, \eta)$ in the oblate spheroidal coordinate are related to the cylindrical coordinate $(\mathrm{r}, \mathrm{z})$ through the relationships of $r=b \sqrt{\left(1+\sigma^{2}\right)\left(1-\eta^{2}\right)}$ and $\mathrm{z}=\mathrm{b} \sigma \eta$, in which $\mathrm{b}$ is the half inter-focal length. The angle variable $\Theta$ is related to $\eta$ as $\eta=\cos \Theta$ and $\gamma$ is the half-aperture angle. The coefficient $\varepsilon$ is set at $1 / 2 \mathrm{~kb}$ and $\mathrm{E}$ is a function of $\sigma$ and $\Theta ; \tau_{\mathrm{s}}$ is the spherically retarded time near the source; $\tau_{\mathrm{p}}$ is the retarded time for planar waves near the focus; $\bar{p}=p / p_{0}$ is the normalized sound pressure with $\mathrm{p}_{0}$ as the initial amplitude of sound pressure on the surface of the source; $\alpha$ is the sound attenuation coefficient; $l_{D}$ is shock formation distance for a planar wave, which is related to the nonlinear coefficient $\beta$ of the medium. If $\beta=0$, the SBE model can be used to predict linear fields.

In the frequency domain, the solutions of Eq. (1) and Eq. (2) are represented by the following Fourier series expansions, respectively [10],

$$
\bar{p}= \begin{cases}\sum_{n=1}^{\infty}\left[g_{n}^{(s)} \sin \left(n \tau_{s}\right)+h_{n}^{(s)} \cos \left(n \tau_{s}\right)\right] & \left(\sigma<\sigma_{0}<0\right) \\ \sum_{n=1}^{\infty}\left[g_{n}^{(p)} \sin \left(n \tau_{p}\right)+h_{n}^{(p)} \cos \left(n \tau_{p}\right)\right] & \left(\sigma \geq \sigma_{0}, \sigma_{0}<0\right)\end{cases}
$$

where $g_{n}{ }^{(s)}, h_{n}{ }^{(s)}, g_{n}{ }^{(p)}$ and $h_{n}{ }^{(p)}$ are amplitude components of the n-th harmonic and are functions of the spatial variables $\boldsymbol{\sigma}$ and $\Theta$.

Substituting Eq. (3) to in Eqs. (1) and (2), we obtain the following two set of coupled nonlinear partial differential equations in the amplitudes of the various Fourier components.

$$
\left\{\begin{array}{l}
\frac{\partial g_{n}}{\partial \sigma}+\frac{1}{2} \frac{\sin (2 \theta)}{\sigma\left(1+\sigma^{2}\right)} \frac{\partial g_{n}}{\partial \theta}+\frac{\varepsilon \sqrt{\sigma^{2}+\sin ^{2} \theta}}{n \cdot \sigma\left(1+\sigma^{2}\right)}\left(\frac{\partial^{2} h_{n}}{\partial \theta^{2}}+\cot \theta \frac{\partial h_{n}}{\partial \theta}\right)+\frac{E}{\sigma} g_{n}= \\
\quad=-\frac{\sqrt{\sigma^{2}+\sin ^{2} \theta}}{\sigma}\left\{-\alpha \cdot b \cdot n^{2} g_{n}+\frac{b}{2 l_{D}} n\left[\frac{1}{2} \sum_{p=1}^{n-1}\left(g_{p} g_{n-p}-h_{p} h_{n-p}\right)-\sum_{p=n+1}^{\infty}\left(g_{p-n} g_{p}+h_{p-n} h_{p}\right)\right]\right\} \\
\frac{\partial h_{n}}{\partial \sigma}+\frac{1}{2} \frac{\sin (2 \theta)}{\sigma\left(1+\sigma^{2}\right)} \frac{\partial h_{n}}{\partial \theta}-\frac{\varepsilon \sqrt{\sigma^{2}+\sin ^{2} \theta}}{n \cdot \sigma\left(1+\sigma^{2}\right)}\left(\frac{\partial^{2} g_{n}}{\partial \theta^{2}}+\cot \theta \frac{\partial g_{n}}{\partial \theta}\right)+\frac{E}{\sigma} h_{n}= \\
=-\frac{\sqrt{\sigma^{2}+\sin ^{2} \theta}}{\sigma}\left\{-\alpha \cdot b \cdot n^{2} h_{n}+\frac{b}{2 l_{D}} n\left[\frac{1}{2} \sum_{p=1}^{n-1}\left(h_{p} g_{n-p}+g_{p} h_{n-p}\right)+\sum_{p=n+1}^{\infty}\left(h_{p-n} g_{p}-g_{p-n} h_{p}\right)\right]\right\}
\end{array}\right.
$$




$$
\left\{\begin{array}{l}
\frac{\partial g_{n}}{\partial \sigma}-\frac{\sigma \sin \theta}{\left(1+\sigma^{2}\right)} \frac{\partial g_{n}}{\partial \theta}-\frac{\varepsilon(2-\cos \theta)}{n \cdot\left(1+\sigma^{2}\right)}\left(\frac{\partial^{2} h_{n}}{\partial \theta^{2}}+\cot \theta \frac{\partial h_{n}}{\partial \theta}\right)= \\
\quad=\left\{-\alpha \cdot b \cdot n^{2} g_{n}+\frac{b}{2 l_{D}} n\left[\frac{1}{2} \sum_{p=1}^{n-1}\left(g_{p} g_{n-p}-h_{p} h_{n-p}\right)-\sum_{p=n+1}^{\infty}\left(g_{p-n} g_{p}+h_{p-n} h_{p}\right)\right]\right\} \quad\left(\sigma \geq \sigma_{0}, \sigma_{0}<0\right) \\
\frac{\partial h_{n}}{\partial \sigma}-\frac{\sigma \sin \theta}{\left(1+\sigma^{2}\right)} \frac{\partial h_{n}}{\partial \theta}+\frac{\varepsilon(2-\cos \theta)}{n \cdot\left(1+\sigma^{2}\right)}\left(\frac{\partial^{2} g_{n}}{\partial \theta^{2}}+\cot \theta \frac{\partial g_{n}}{\partial \theta}\right)= \\
\quad=\left\{-\alpha \cdot b \cdot n^{2} g_{n}+\frac{b}{2 l_{D}} n\left[\frac{1}{2} \sum_{p=1}^{n-1}\left(h_{p} g_{n-p}+g_{p} h_{n-p}\right)+\sum_{p=n+1}^{\infty}\left(h_{p-n} g_{p}-g_{p-n} h_{p}\right)\right]\right\} E
\end{array}\right.
$$

It is practicable to obtain the solution of the $\mathrm{n}$-th harmonic component using an implicit backward finite difference method.

Now let the source be excited simultaneously at the two frequencies $f_{1}$ and $f_{2}$. We impose periodicity in the boundary conditions on the source, and the two frequencies must therefore be commensurable. This means that there exist $f$ such that

$$
f_{1}=\mathrm{N}_{1} f \text { and } f_{2}=\mathrm{N}_{2} f
$$

where $\mathrm{N}_{1}$ and $\mathrm{N}_{2}$ are integers greater than unity. Let $f$ denote the largest possible frequency that satisfies these conditions. We refer to $f$ as the basic frequency and to $f_{-}=\left|f_{1}-f_{2}\right|$ as the difference frequency. Also, the center frequency $f_{m}=\left(f_{1}+f_{2}\right) / f$ and the downshift ratio $f_{m} / f_{-}$ are introduced.

Consequently, boundary conditions at the source in the SBE system yield the following relation:

$$
\left.\bar{p}\right|_{\sigma=-\sigma \max }=U_{1}(\theta) \sin \left(N_{1} \tau_{s}+\phi\right)+U_{2}(\theta) \sin \left(N_{2} \tau_{s}\right)
$$

where $U_{1}(\Theta)$ and $U_{2}(\Theta)$ are the pressure distribution function on the surface of the source, both $\mathrm{N}_{1}$ and $\mathrm{N}_{2}$ are integer greater than unity, and $\phi$ is the initial phase. The value of initial phase does not play an important role in the parametric amplification under the condition of $\mathrm{N}_{1} \geq 3$ and $\mathrm{N}_{2} \geq 3$, which the difference frequency is essentially independent of the variation of $\phi$ [9]. Therefore, the zero phase of $\phi$ is used for the sake of convenience.

Thus, the initial values of $g_{n}{ }^{(s)}$ and $h_{n}{ }^{(s)}$ at $\sigma=-\sigma_{\max }$ are given through the boundary condition of Eq. (7):

$$
\left\{\begin{array}{l}
g_{N_{1}}^{(s)}=U_{1}(\theta) \cos \phi \\
h_{N_{1}}^{(s)}=0 \\
g_{N_{2}}^{(s)}=U_{2}(\theta) \\
h_{N_{2}}^{(s)}=U_{1}(\theta) \sin \phi \\
g_{n}^{(s)}=h_{n}^{(s)}=0, \quad n \neq N_{1}, N_{2}
\end{array}\right.
$$

In this section we present numerical results for the acoustic field from a strongly focusing source with uniform amplitude distribution described by

$$
U_{1}(\theta)=U_{2}(\theta)=\left\{\begin{array}{cc}
1 & (0 \leq \theta \leq \gamma) \\
0 & (\text { others })
\end{array}\right.
$$

Then, the pressure amplitudes at $\boldsymbol{\sigma}=-\boldsymbol{\sigma}_{\max }$ are obtained by $\sqrt{g_{n}^{(s)^{2}}+h_{n}^{(s)^{2}}}$

\section{NUMERICAL EXAMPLES}

To examine the validity of SBE for parametric focusing source with a concave spherical source, the effects of focusing are carried out for the primary, difference and sum frequency waves with $f_{\mathrm{m}} / f_{-}=5.5, \mathrm{p}_{0}=100 \mathrm{Kpa}, \mathrm{d}=10 \mathrm{~cm}, \gamma=30^{\circ}$. In addition, degassed water, with sound velocity $c_{0}=1500$ $\mathrm{m} / \mathrm{s}$, density $\mathrm{p}_{\mathrm{o}}=1000 \mathrm{~kg} / \mathrm{m}^{3}$, nonlinear coefficient $\beta=3.5$, and attenuation coefficient $\alpha=0.0253 \mathrm{~Np} / \mathrm{m}$ at $1 \mathrm{MHz}$ with frequency dependence $f^{2}$, is used as the propagation medium in the simulation. Since it is not easy to solve the SBE equation analytically, numerical computation is performed by means of an implicit backward finite difference method. For ensuring reasonable accuracy, we establish 600 grid points from 0 to $\Pi / 2$ on the $\Theta$ axis, and thus the step size of $\Theta$ is $2.618 \times 10^{-3} \mathrm{rad}$. The transition location is numerically determined to be a reasonable choice for $\boldsymbol{\sigma}_{0}=-1.0[7]$.

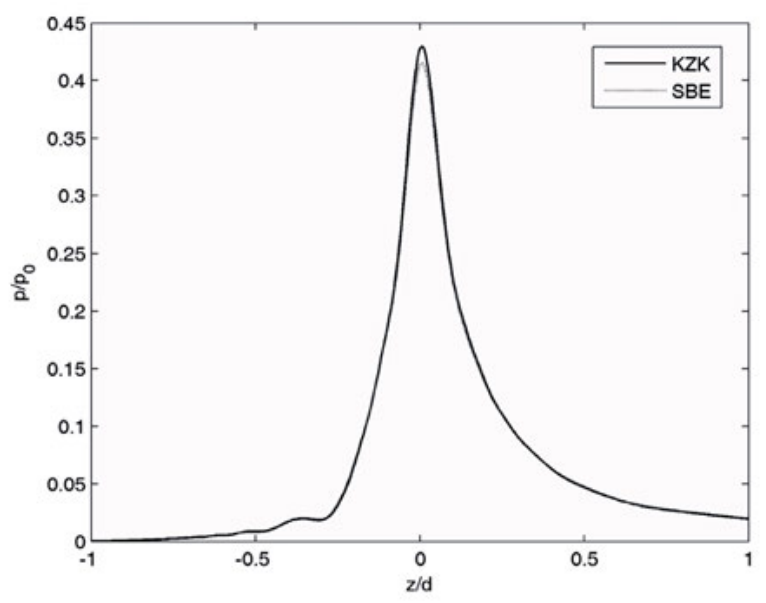

Fig. 2a. On-axis Comparison of pressure amplitude between the SBE model and KZK model for difference frequency wave 


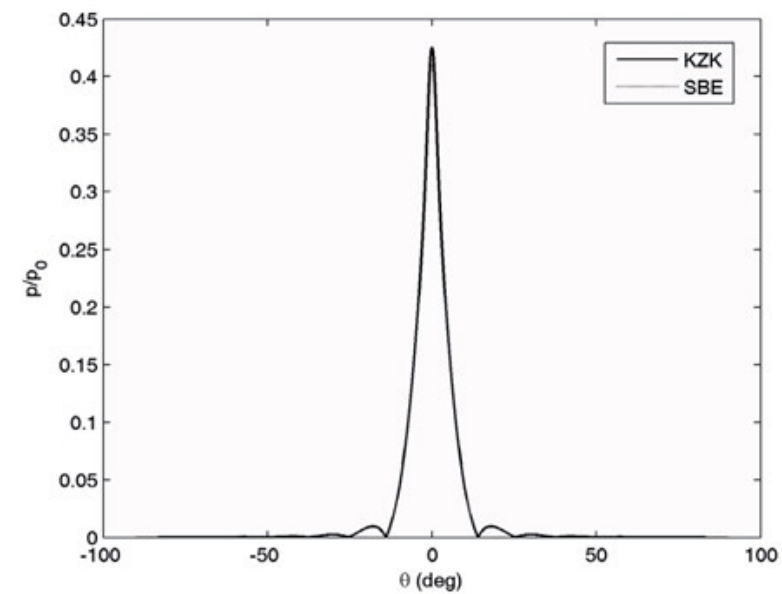

Fig. 2b. Beam patterns in the focal plane Comparison of pressure amplitude between the SBE model and KZK model for difference frequency wave

An interesting comparison is shown in Fig. 2 for the SBE and KZK solutions with the difference frequency wave. It can be found that the pressure amplitude from SBE is slightly smaller than that from KZK model in the focal region and the maximum deviation is less than $3 \%$, but both models are in good agreement in the axial distribution and beam patterns. Moreover, previous studies [3] validated effectiveness of KZK model for a parametric focusing source. Consequently, the focused parametric model of SBE is also good valid for a parametric focusing source on a concave spherical surface with a large aperture angle in the acoustic field.

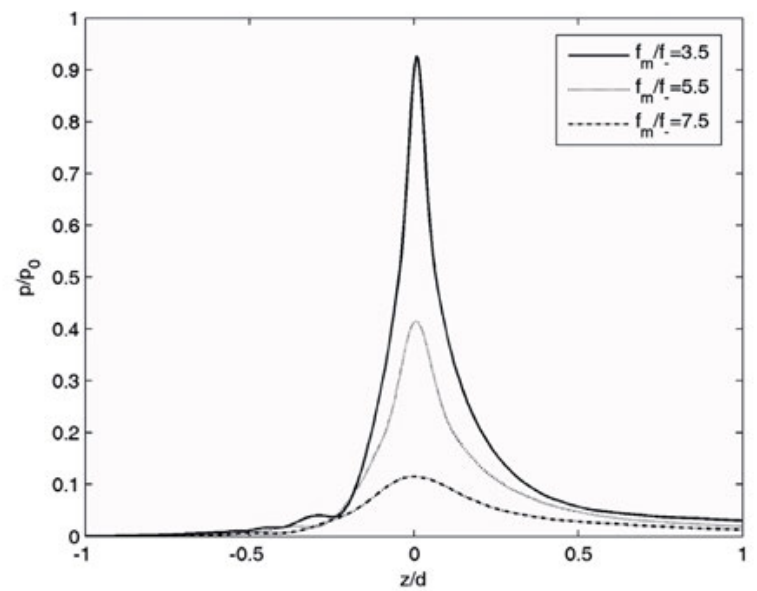

Fig. 3a. On-axis Comparison of pressure amplitude for difference frequency wave using SBE model with three different of downshift ratios

For three different of downshift ratios (e.g. $f_{\mathrm{m}} / f_{-}=3.5,5.5$, and 7.5), the corresponding propagation curves and beam patterns for the difference frequency wave are shown in Fig.3. With the downshift ratio $f_{\mathrm{m}} / f_{-}$increasing, the pressure amplitude along the axis is gradually decreasing, while the angle of $-3 \mathrm{~dB}$ beam width is evidently broadening in the focal region. Also, the pressure amplitude varies very slowly whether along the axis or across the axis. This means that the increasing of downshift ratio $f_{\mathrm{m}} / f_{-}$weakens the efficiency of difference frequency generation.

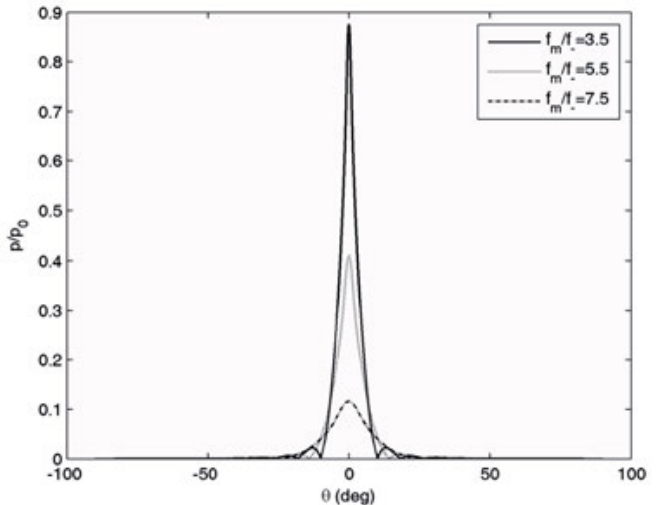

Fig. 3b. Beam patterns in the focal plane Comparison of pressure amplitude for difference frequency wave using SBE model with three different of downshift ratios

Figure 4 shows propagation curve and beam patterns for the difference wave with three different of half-aperture angles (e.g. $\gamma=20^{\circ}, 30^{\circ}$, and $40^{\circ}$ ) and a fixed downshift ratio $f_{\mathrm{m}} / f_{-}=5.5$. The larger the half-aperture angle $\gamma$, the higher is the pressure amplitude along the axis, and the shorter is the angle of $-3 \mathrm{~dB}$ beam width in the focal region.

From Figs. 3 and 4, it can be implemented by decreasing of the downshift ratio $f_{\mathrm{m}} / f_{-}$and increasing of the half-aperture angle $\gamma$ for high directivity and resolution, good focused ability and penetrative performance.

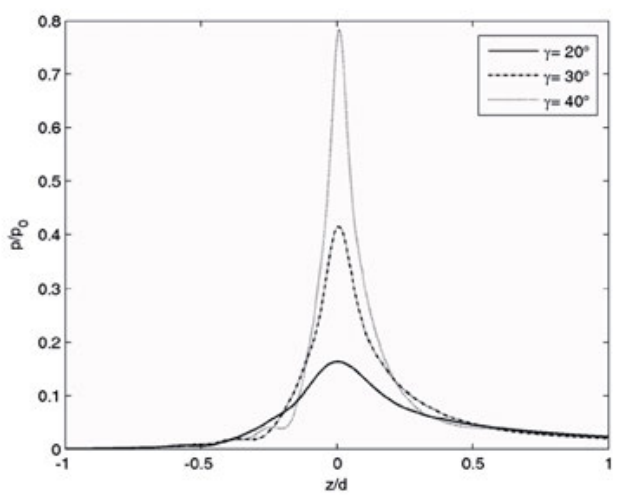

Fig. 4a. On-axis Comparison of pressure amplitude for difference frequency wave using SBE model with three different of half-aperture angle

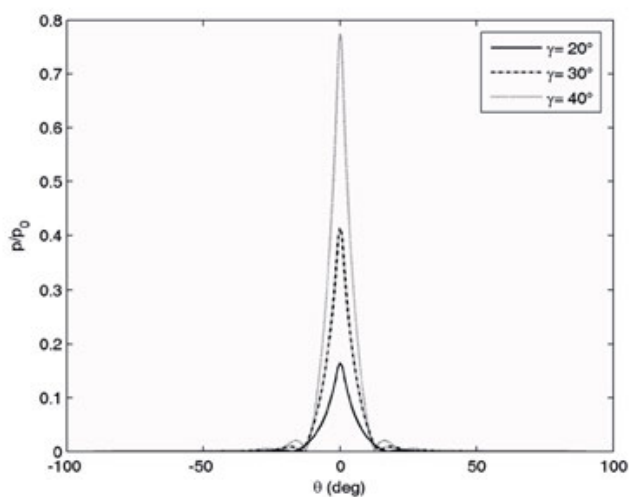

Fig. 4b. Beam patterns in the focal plane Comparison of pressure amplitude for difference frequency wave using SBE model with three different of halfaperture angle 


\section{CONCLUSION}

In conclusion, this study proposes source boundary conditions using SBE for a focused parametric source on a concave spherical source. The pilot study of parametric effects by SBE model is implemented, numerical results of which are good agree with that of KZK model. The simulations show that the difference frequency radiation is effectively focused, in that the $-3 \mathrm{~dB}$ beam width became quite narrow in the focal plane. In addition, with decreasing the downshift ratio and increasing the half-aperture angle, it can be obtained that high directivity and resolution, good focused ability and penetrative performance. It is really benefit to distinguishing seabed properties and detecting buried object. Compared with KZK model, a focused parametric model of SBE lays a theoretical basis for the study of parametric effects under high frequencies and large aperture angles.

\section{ACKNOWLEDGMENTS}

The authors thank Prof. Tomoo Kamakura, the university of Electro-Communication, Japan, for his useful help in solving the SBE programming.

This paper was supported by the National Natural Science Foundation of China under Grant No. 51209133, Program for Innovative Research Team in Shanghai Maritime University, Shanghai Municipal Education Commission First-class Disciplines Project and Innovation Project of Shanghai Graduate Education (No. 20131129).

\section{REFERENCES}

1. D Boulinguez, A Quinquis, 3-D underwater object recognition. IEEE Journal of Oceanic Engineering, 2002, 27(4), 814-829.

2. E Kozaczka, G Grelowska, S Kozaczka, and W Szymczak, Detection of Objects Buried in the Sea Bottom with the Use of Parametric Echosounder. Archives of Acoustics, 2013, 38(1), 99-104.

3. J. Naze Tjфtta, S. Tjфtta and E.H. Vefring, Effects of focusing on the nonlinear interaction between two collinear finite amplitude sound beams, J.Acoust.Soc.Am. 1991, 89 (3), 1017-1027.

4. J. Naze Tjфtta, S. Tjфtta, and E.H. Vefring, Propagation and interaction of two collinear finite amplitude sound beams, J.Acoust.Soc.Am. 1990, 88 (6), 2859-2869.

5. J. Wunderlich, S. Müller, High-resolution sub-bottom profiling using parametric acoustics. International Ocean Systems, 2003, 7(4), 6-11.

6. P.J. Westervelt, Parametric Acoustic Array. J.Acoust.Soc. Am. 1963, 35(4), 535-537.
7. R.M. Xia, W.D. Shou, G.P. Chen and M,D. Zhang, The further study of the spheroidal beam equation for focused finite-amplitude sound beams, J.Comput. Acoust. 2003, 11 (1), 47-53.

8. S.Y. Qian, T. Kamakura and M. Akiyama, Simulation of sound field in a tissue medium generated by a concave spherically annular transducer, Ultrasonics, 2006, 44 (1), 271-274

9. T. Kamakura, N. Hamada, K. Aoki, and Y. Kumamoto, Nonlinearly generated spectral components in the nearfield of a directive sound source. J.Acoust.Soc.Am. 1989, 85 (6), 2331-2337.

10. T. Kamakura, T. Ishiwata and K. Matsuda, Model equation for stringly focused finite-amplitude sound beams, J.Acoust.Soc.Am. 2000, 107 (6), 3035-3046.

\section{CONTACT WITH AUTHOR}

\author{
Lili Yu \\ llyu@shmtu.edu.cn \\ Chaojian Shi \\ Department of Navigation Technology \\ Shanghai Maritime University \\ 200136 Shanghai \\ China
}

Junhua Li

College of Ocean Science and Engineering Shanghai Maritime University 200136 Shanghai China 